\title{
Dynamic Analysis for a Kaldor-Kalecki Model of Business Cycle with Time Delay and Diffusion Effect
}

\author{
Wenjie Hu $\mathbb{D}^{1,2}$ Hua Zhao $\mathbb{D}^{1,3}$ and Tao Dong ${ }^{4}$ \\ ${ }^{1}$ College of Economics and Business Administration, Chongqing University, Chongqing 400030, China \\ ${ }^{2}$ College of Economics and Management, Chongqing University of Posts and Telecommunications, Chongqing 400065, China \\ ${ }^{3}$ School of Management, Chongqing Technology and Business University, Chongqing 4000067, China \\ ${ }^{4}$ College of Electronics and Information Engineering, Southwest University, Chongqing 400715, China
}

Correspondence should be addressed to Hua Zhao; zhaohua@cqu.edu.cn

Received 5 April 2017; Accepted 6 December 2017; Published 9 January 2018

Academic Editor: Dimitri Volchenkov

Copyright (c) 2018 Wenjie Hu et al. This is an open access article distributed under the Creative Commons Attribution License, which permits unrestricted use, distribution, and reproduction in any medium, provided the original work is properly cited.

\begin{abstract}
The dynamics behaviors of Kaldor-Kalecki business cycle model with diffusion effect and time delay under the Neumann boundary conditions are investigated. First the conditions of time-independent and time-dependent stability are investigated. Then, we find that the time delay can give rise to the Hopf bifurcation when the time delay passes a critical value. Moreover, the normal form of Hopf bifurcations is obtained by using the center manifold theorem and normal form theory of the partial differential equation, which can determine the bifurcation direction and the stability of the periodic solutions. Finally, numerical results not only validate the obtained theorems, but also show that the diffusion coefficients play a key role in the spatial pattern. With the diffusion coefficients increasing, different patterns appear.
\end{abstract}

\section{Introduction}

Recently, business cycle, as one of the important economic phenomena, has received attractive attentions due its widely application in many fields such as economic decisions, macroeconomic regulation, and market regulation [1-8]. In order to understand the mechanisms of business cycle, many models are proposed. One of the most famous business cycle models is the Kaldor-Kalecki business cycle $[9,10]$, which is described as

$$
\begin{aligned}
& \frac{d Y(t)}{\partial t}=\alpha[I(Y(t), K(t))-\gamma Y(t)] \\
& \frac{d K(t)}{\partial t}=I(Y((t)), K(t))-q K(t),
\end{aligned}
$$

where $Y(t)$ is the gross product, $K(t)$ is the capital stock at time, $\alpha$ is the adjustment coefficient in the goods market, $q$ is the depreciation rate of the capital stock, $\gamma$ represents the propensity to save, and $I(Y(t), K(t))$ is the investment. Under this model, the dynamic behaviors are widely studied such as stability, Hopf bifurcation, codimension-two bifurcation, and chaos [9-15].

It is well known that diffusion effects of economic activities are widespread phenomenon that existed all over the world. As a result of the impact of the growth pole, the diffusion effects are the main interactions in economic activities. So, the diffusion effect should be considered in the business cycle model. However, to the best of our knowledge, there are very few works on this field. Inspired by the observation, in this paper, based on the Kaldor-Kalecki model, we propose a novel business cycle with diffusion effect and time delay under the Neumann boundary conditions, which is as follows:

$$
\begin{aligned}
\frac{\partial Y(t, x)}{\partial t}= & d_{1} \Delta Y(t, x) \\
& +\alpha[I(Y(t, x))-\beta K(t, x)-\gamma Y(t, x)] \\
\frac{\partial K(t, x)}{\partial t}= & d_{2} \Delta K(t, x)+I(Y((t-\tau), x)) \\
& -(q+\beta) K(t, x),
\end{aligned}
$$


with its initial and boundary conditions given as follows:

$$
\begin{array}{r}
\frac{\partial u_{1}}{\partial x}=\frac{\partial v_{1}}{\partial x}=\frac{\partial u_{2}}{\partial x}=\frac{\partial v_{2}}{\partial x}=0, \quad t>0, x \in \partial \Omega \\
u_{i}(t, x)=\phi_{i}(t, x), \quad i=1,2, t \in[-\tau, 0], x \in \bar{\Omega} \\
v_{i}(t, x)=\phi_{i}(t, x), \quad i=1,2, t \in[-\tau, 0], \quad x \in \bar{\Omega},
\end{array}
$$

where $\alpha>0, \beta>0, q \in(0,1), \gamma \in(0,1)$, and $\Omega$ is the market capacity. There are three contributions of this paper:

(1) Based on the Kaldor-Kalecki model, we propose a novel business cycle under the Neumann boundary conditions. Our model is a spatial-temporal model, which is more general than the existing models.

(2) The time-independent and time-dependent stability are investigated. Moreover, the conditions of the Hopf bifurcation are obtained.

(3) It is found that the diffusion coefficients play a key role in the spatial pattern. With the diffusion coefficients increasing, different patterns appear.

The rest paper is organized as follows. In Section 2, the time-independent stability, time-dependent stability, and the existence of Hopf bifurcation are obtained. In Section 3, the normal form of Hopf bifurcation is obtained. In Section 4, numerical results are given to validate the obtained theorems.

\section{Local Stability and Hopf Bifurcation Analysis}

Let $\left(Y^{*}, K^{*}\right)$ be an equilibrium of $(2)$ and $u_{1}(t, x)=Y(t, x)-$ $Y^{*}, u_{2}(t, x)=K(t, x)-K^{*}, i(u(t, x))=I(u(t, x))-I^{*}$, and $s=S-S^{*} ;(2)$ can be rewrite as

$$
\begin{aligned}
\frac{\partial u_{1}(t, x)}{\partial t}= & d_{1} \Delta u_{1}(t, x) \\
& +\alpha\left[i\left(u_{1}(t, x)\right)-\beta u_{2}(t, x)-\gamma u_{1}(t, x)\right] \\
\frac{\partial u_{2}(t, x)}{\partial t}= & d_{2} \Delta u_{2}(t, x)+i\left(u_{1}((t-\tau), x)\right) \\
& -(q+\beta) u_{2}(t, x) .
\end{aligned}
$$

Taking the Taylor expansion of $i$ at 0 yields

$$
i(u)=c_{1} u+c_{2} u^{2}+c_{3} u^{3}+\cdots,
$$

where $c_{1}=i^{\prime}(0), c_{2}=(1 / 2) i^{\prime \prime}(0)$, and $c_{3}=(1 / 3 !) i^{\prime \prime \prime}(0)$.

The linear parts of (4) are as follows:

$$
\begin{aligned}
\frac{\partial u_{1}(t, x)}{\partial t}= & d_{1} \Delta u_{1}(t, x)+\alpha\left(c_{1}-\gamma\right) u_{1}(t, x) \\
& -\alpha \beta u_{2}(t, x) \\
\frac{\partial u_{2}(t, x)}{\partial t}= & d_{2} \Delta u_{2}(t, x)+c_{1} u_{1}((t-\tau), x) \\
& -(q+\beta) u_{2}(t, x) .
\end{aligned}
$$

Then, the characteristic of (6) is

$$
\lambda y-D \Delta y-L\left(e^{\lambda} y\right)=0, \quad y \in \operatorname{dom}(\Delta) \backslash\{0\} .
$$

Following the method of [16], we define $-k^{2}\left(k \in \mathfrak{R}_{0}=\right.$ $\{0,1,2, \ldots\})$ as the eigenvalue of $\Delta$ under the Neumann boundary conditions on the $X$. Let $\beta_{k}^{1}=\left(\gamma_{k}, 0,\right)^{T}, \beta_{k}^{2}=$ $\left(0, \gamma_{k}\right)^{T}$ be the corresponding eigenvectors, where $\gamma_{k}=$ $\cos (k x), k=0,1,2, \ldots$ We use $\left\{\beta_{k}^{1}, \beta_{k}^{2}\right\}_{k=0}^{\infty}$ to construct a basis of the phase space $X$. Then, $y$ can be expanded in the following form of Fourier on $X$ :

$$
\begin{gathered}
y=\sum_{k=0}^{\infty} Y_{k}^{T}\left(\begin{array}{c}
\beta_{k}^{1} \\
\beta_{k}^{2} \\
\beta_{k}^{3} \\
\beta_{k}^{4}
\end{array}\right), \\
Y_{k}=\left(\begin{array}{c}
\left\langle y, \beta_{k}^{1}\right\rangle \\
\left\langle y, \beta_{k}^{2}\right\rangle \\
\left\langle y, \beta_{k}^{3}\right\rangle \\
\left\langle y, \beta_{k}^{4}\right\rangle
\end{array}\right)^{T}
\end{gathered}
$$

Then, we have

$$
L\left(\phi^{T}\left(\begin{array}{l}
\beta_{k}^{1} \\
\beta_{k}^{2} \\
\beta_{k}^{3} \\
\beta_{k}^{4}
\end{array}\right)\right)=L(\phi)^{T}\left(\begin{array}{c}
\beta_{k}^{1} \\
\beta_{k}^{2} \\
\beta_{k}^{3} \\
\beta_{k}^{4}
\end{array}\right), \quad k \in N_{0} .
$$

By (7)-(9), we can obtain

$$
\begin{gathered}
\operatorname{det}\left(\left[\begin{array}{cc}
\lambda+d_{1} k^{2} & 0 \\
0 & \lambda+d_{2} k^{2}
\end{array}\right]\right. \\
\left.-\left[\begin{array}{cc}
\alpha\left(c_{1}-\gamma\right) & -\alpha \beta \\
c_{1} e^{-\lambda \tau} & -(q+\beta)
\end{array}\right]\right)=0 .
\end{gathered}
$$

The characteristic equation of (10) is as follows:

$$
\left(\begin{array}{cc}
\lambda+d_{1} k^{2}-\alpha\left(c_{1}-\gamma\right) & \alpha \beta \\
-c_{1} e^{-\lambda \tau} & \lambda+d_{2} k^{2}+(q+\beta)
\end{array}\right)=0 .
$$

The characteristic equation of (11) is

$$
F(\lambda)=\lambda^{2}+\varphi_{1}(k) \lambda+\varphi_{2}(k)+\alpha \beta c_{1} e^{-\lambda \tau},
$$

where

$$
\begin{aligned}
\varphi_{1}(k)= & \left(d_{1}+d_{2}\right) k^{2}+q+\beta-\alpha\left(c_{1}-\gamma\right) \\
\varphi_{2}(k)= & d_{1} d_{2} k^{4}+\left(d_{1} q+d_{1} \beta-d_{2}\left(c_{1}-\gamma\right) \alpha\right) k^{2} \\
& -\left(c_{1}-\gamma\right)(q \alpha+\alpha \beta) .
\end{aligned}
$$


Lemma 1. If $c_{1}<\gamma$ holds, the positive equilibrium of system (2) is locally stable at $\tau=0$.

Proof. For $\tau=0$, (12) can be rewrite as $F(\lambda)=\lambda^{2}+\varphi_{1}(k) \lambda+$ $\varphi_{2}(k)+\alpha \beta c_{1}$. As $c_{1}<\gamma, \alpha>0, \beta>0, q \in(0,1)$, and $\gamma \in(0,1)$, one can obtain $\forall k \in N, \varphi_{1}(k)>0$ and $\varphi_{2}(k)+\alpha \beta c_{1}>$ $\alpha\left(\gamma-c_{1}\right) q+\gamma q>0$ which means the roots of (12) have negative part. Therefore, the positive equilibrium of system (2) is locally stable at $\tau=0$. The proof is completed.

Substituting $\lambda=i \omega$ into (12) and separating real parts and imaginary parts yield

$$
\begin{aligned}
& \alpha \beta c_{1} \cos \omega \tau=\omega^{2}-\varphi_{2}(k) \\
& \alpha \beta c_{1} \sin \omega \tau=\varphi_{1}(k) \omega .
\end{aligned}
$$

According to $\sin ^{2} \omega \tau+\cos ^{2} \omega \tau=1$, from (14), we have

$$
\begin{aligned}
F(\omega) & =\omega^{4}+\left(\varphi_{1}^{2}(k)-2 \varphi_{2}(k)\right) \omega^{2}+\varphi_{2}^{2}(k)-\left(\alpha \beta c_{1}\right)^{2} \\
& =0
\end{aligned}
$$

By simple calculation, we have

$$
\begin{aligned}
& \varphi_{1}^{2}(k)-2 \varphi_{2}(k) \\
& =\left(\alpha\left(\gamma-c_{1}\right)+d_{1} k^{2}\right)^{2}+\left(q+\beta+d_{2} k^{2}\right)^{2}>0 \\
& \varphi_{2}^{2}(k)-\left(\alpha \beta c_{1}\right)^{2} \\
& \quad=\left(\alpha\left(\gamma-c_{1}\right)+d_{1} k^{2}\right)^{2}\left(q+\beta+d_{2} k^{2}\right)^{2}-\left(\alpha \beta c_{1}\right)^{2} .
\end{aligned}
$$

As $d_{i}>0, i=1,2$, one can obtain

$$
\begin{aligned}
& \varphi_{2}^{2}(k)-\left(\alpha \beta c_{1}\right)^{2} \\
& \quad=\left(\alpha\left(\gamma-c_{1}\right)+d_{1} k^{2}\right)^{2}\left(q+\beta+d_{2} k^{2}\right)^{2}-\left(\alpha \beta c_{1}\right)^{2} \\
& \quad>\alpha^{2}\left(\gamma-c_{1}\right)^{2}(q+\beta)^{2}-\left(\alpha \beta c_{1}\right)^{2}
\end{aligned}
$$

It is easy to see if $\left(\gamma-c_{1}\right)(q+\beta)-\left(\beta c_{1}\right)>0,(15)$ has no positive roots. Combining with the Lemma 1 , we have the following theorem.

Theorem 2. If $c_{1}<\gamma$ and $\left(\gamma-c_{1}\right)(q+\beta)-\left(\beta c_{1}\right)>0$, the positive equilibrium of system (2) is locally stable for any $\tau>0$.

In the following, one investigates the conditions of Hopf bifurcation of (2). By (14), one can obtain

$$
\begin{aligned}
& \cos \omega \tau=\frac{\omega^{2}-\varphi_{2}(k)}{\alpha \beta c_{1}} \\
& \sin \omega \tau=\frac{\varphi_{1}(k) \omega}{\alpha \beta c_{1}} .
\end{aligned}
$$

By simple calculation, one has

$$
\begin{aligned}
& \frac{d \omega}{d k} \\
& =-\frac{\left(4 b_{1} k^{3}+2 b_{2} k+b_{3}\right) \omega^{2}+8 b_{4} k^{7}+6 b_{5} k^{5}+4 b_{6} k^{3}+2 b_{7} k}{4 \omega^{3}+2\left(b_{1} k^{4}+b_{2} k^{2}+b_{3}\right) \omega}
\end{aligned}
$$$$
<0 \text {, }
$$

where

$$
\begin{aligned}
& b_{1}=a_{5}^{2}-2 a_{1}, \\
& b_{2}=2\left(a_{5} a_{6}-a_{2}\right), \\
& b_{3}=a_{6}^{2}+2 a_{3}, \\
& b_{4}=a_{1}^{2}, \\
& b_{5}=2 a_{1} a_{2}, \\
& b_{6}=a_{2}^{2}-2 a_{1} a_{3}, \\
& b_{7}=-2 a_{2} a_{3}, \\
& b_{8}=a_{3}^{2}-a_{4}^{2}, \\
& a_{1}=d_{1} d_{2}, \\
& a_{2}=d_{1} q+d_{1} \beta-d_{2} \alpha\left(c_{1}-\gamma\right), \\
& a_{3}=\alpha(q+\beta)\left(c_{1}-\gamma\right), \\
& a_{4}=\alpha \beta c_{1}, \\
& a_{5}=d_{1}+d_{2}, \\
& a_{6}=q+\beta-\alpha\left(c_{1}-\gamma\right) .
\end{aligned}
$$

If $c_{1}<\gamma$, by simple calculation, one can obtain $b_{i}>0, i=$ $1,2, \ldots, 7$, and then one has $d \omega / d k<0$. From (18), one has

$$
\tan \omega \tau=\frac{\varphi_{1}(k) \omega}{\omega^{2}-\varphi_{2}(k)}
$$

Taking the derivative of $k$ in (21), one can obtain

$$
\begin{aligned}
\frac{d \tau}{d k} & =-\left(\frac{1}{1+e_{0}}\left(e_{1} \omega+\frac{e_{2} k^{6}+e_{3} k^{4}+e_{4} k^{2}+e_{5}}{\omega}\right)\right. \\
& \left.+\frac{\tau}{\omega}\right) \frac{d \omega}{d k}+\frac{1}{1+e_{0}}\left(e_{6} \omega^{2}+e_{7}\right),
\end{aligned}
$$


where

$$
\begin{aligned}
& e_{0}=\left(\frac{\left(a_{5} k^{2}+a_{6}\right) \omega}{\omega^{2}-a_{1} k^{4}-a_{2} k^{2}+a_{3}}\right)^{2}>0, \\
& e_{1}=a_{5} k^{2}+a_{6}>0, \\
& e_{2}=a_{1} a_{5}>0, \\
& e_{3}=a_{2} a_{5}+a_{1} a_{6}, \\
& e_{4}=a_{2} a_{6}-a_{3} a_{5}, \\
& e_{5}=-a_{3} a_{6}, \\
& e_{6}=2 a_{5} k, \\
& e_{7}=2 a_{1} a_{5} k^{5}+4 a_{1} a_{6} k^{3}+2\left(a_{2} a_{6}+a_{3} a_{5}\right) k .
\end{aligned}
$$

If $c_{1}<\gamma$, one can obtain $a_{3}<0$; by simple calculation, one can get $e_{i}>0, i=0,1, \ldots, 7$. Combining with $d \omega / d k<0$, one has

$$
\frac{d \tau}{d k}>0
$$

which means $\tau$ increase with the increasing of $k$. So, $\tau_{0}$ must exist in $k=0$. Now, one considers the case $k=0$. Let $z=\omega^{2}$; (18) with $k=0$ is as follows:

$$
\begin{aligned}
F(z)= & z^{2}+\left(\alpha^{2}\left(\gamma-c_{1}\right)^{2}+(q+\beta)^{2}\right) z \\
& +\alpha^{2}\left(\gamma-c_{1}\right)^{2}(q+\beta)^{2}-\left(\alpha \beta c_{1}\right)^{2} .
\end{aligned}
$$

Since $\alpha^{2}\left(\gamma-c_{1}\right)^{2}+(q+\beta)^{2}>0,(25)$ has only one positive root of $(25)$ if $\left(\gamma-c_{1}\right)(q+\beta)-\left(\beta c_{1}\right)<0$. Let $z$ be the positive root of (25); then one can obtain $\omega=\sqrt{z}$

$$
\tau_{j}^{0}=\frac{1}{\omega} \arctan \frac{\varphi_{1}(0) \omega}{\omega^{2}-\varphi_{2}(0)}+\frac{1}{\omega} j \pi, \quad j=1,2, \ldots
$$

By (26), one can obtain $\tau_{0}=\tau_{0}^{0}$.

Lemma 3 (see $[16,17])$. Consider the exponential polynomial

$$
\begin{aligned}
P(\lambda, & \left.e^{-\lambda \tau}, \ldots, e^{-\lambda \tau m}\right) \\
= & \lambda^{n}+P_{1}^{(0)} \lambda^{n-1}+\cdots+P_{n-1}^{(0)} \lambda+P_{n}^{(0)} \\
& +\left[P_{1}^{(1)} \lambda^{n-1}+\cdots+P_{n-1}^{(1)} \lambda+P_{n}^{(1)}\right] e^{-\lambda \tau}+\cdots \\
& +\left[P_{1}^{(m)} \lambda^{n-1}+\cdots+P_{n-1}^{(m)} \lambda+P_{n}^{(m)}\right] e^{-\lambda \tau m},
\end{aligned}
$$

where $\tau_{i} \geq 0(i=1,2, \ldots, m)$ and $p_{j}^{(i)}(j=1,2, \ldots, m)$ are constants. As $\left(\tau_{1}, \tau_{2}, \ldots, \tau_{m}\right)$ vary, the sum of the order of the zeroes of $P\left(\lambda, e^{-\lambda \tau}, \ldots, e^{-\lambda \tau_{m}}\right)$ in the open right half plane can change only if a zero appears on or crosses the imaginary axis.

Theorem 4. According to Lemmas 1 and 3, one has the following.

If $c_{1}<\gamma$ and $\left(\gamma-c_{1}\right)(q+\beta)-\left(\beta c_{1}\right)<0$ holds, system (2) is asymptotically stable for $\tau \in\left[0, \tau_{0}\right)$. System (2) undergoes a
Hopf bifurcation at the origin when $\tau=\tau_{0}$; that is, system (2) has a branch of periodic solutions bifurcating from the trivial solution near $\tau=\tau_{0}$.

Remark 5. By incorporate diffusion effect into the KaldorKalecki model of business cycle, a novel Kaldor-Kalecki model of business cycle with diffusion effect and time delay is proposed. Our model is a spatial-temporal model, which is more general than the existing business cycle $[8,9]$.

\section{Direction and Stability of the Hopf Bifurcation}

In this section, we give the normal form of Hopf bifurcation of (2) by using the method of $[16,18]$. Let $\vartheta=\tau-\tau_{0}$ and normalize $\tau$ by $t \rightarrow t / \tau$; (2) can be rewritten as

$$
\dot{R}(t)=\tau_{0} D U(t)+L\left(\tau_{0}\right)(U(t))+F(U(t), \vartheta),
$$

where

$$
\begin{aligned}
D & =\operatorname{diag}\left(D_{1}, D_{2}\right), \\
L\left(\tau_{0}\right)(\varphi) & =\tau_{0}\left(B_{0} \varphi(0)+B_{1} \varphi(-\tau)\right), \\
B_{0} & =\left[\begin{array}{cc}
\alpha h & -\alpha \beta \\
0 & -(q+\beta)
\end{array}\right] \\
B_{1} & =\left[\begin{array}{ll}
0 & 0 \\
c_{1} & 0
\end{array}\right] . \\
F(\varphi, \vartheta) & =\vartheta D \Delta \varphi(0)+L(\vartheta)(\varphi)+f^{*}(\varphi, \vartheta) \\
f^{*}(\varphi, \vartheta) & =\left(\tau_{0}+\vartheta\right)\left(\begin{array}{l}
\alpha c_{2} \varphi_{1}^{2}(0)+\alpha c_{3} \varphi_{1}^{3}(0) \\
c_{2} \varphi_{1}^{2}(-1)+c_{3} \varphi_{1}^{3}(-1)
\end{array}\right), \\
c_{1} & =i^{\prime}(0), \\
c_{2} & =\frac{1}{2} i^{\prime \prime}(0), \\
c_{3} & =\frac{1}{3} i^{\prime \prime \prime}(0) .
\end{aligned}
$$

For $\varphi=\left(\varphi_{1}, \varphi_{2}\right)^{T} \in \zeta$, from Theorem 2, we know $\Lambda_{0}\left(-i \omega_{0} \tau_{0}, i \omega_{0} \tau_{0}\right)$ are the eigenvalues of the linear part of (28):

$$
\begin{aligned}
\dot{U}(t) & =\tau_{0} D u(t)+L\left(\tau_{0}\right)(u(t)), \\
\dot{z}(t) & =L\left(\tau_{0}\right)\left(z_{t}\right),
\end{aligned}
$$

where $L\left(\tau_{0}\right)$ is one parameter family of bounded linear operator in $C:=C\left([-1,0] \Re^{2}\right)$ into $\Re^{2}$.

Following the method of [16], we define $\eta(\theta, \tau)$ for $\theta \in$ $[-1,0]$, such that

$$
L^{*}\left(\tau_{0}\right)(\varphi)=\int_{-1}^{0} d \eta\left(\theta, \tau_{0}\right) \varphi(\theta), \quad \varphi \in C,
$$

where

$$
\eta\left(\theta, \tau_{0}\right)=\left(\tau_{0}+\vartheta\right)\left[B_{0} \delta(\theta)+B_{1} \delta(\theta+1)\right] .
$$

$\delta(\theta)$ is Drac-delta function. 
Let $A\left(\tau_{0}\right)$ be the infinitesimal generator of the semigroup induce by the solution of (28) and $A^{*}$ denote the formal adjoint of $A\left(\tau_{0}\right)$ under the bilinear pairing; we have

$$
\begin{aligned}
\langle\psi, \varphi\rangle= & \psi(0) \cdot \varphi(0) \\
& -\int_{\theta=-1}^{0} \int_{\xi=0}^{\theta} \psi(\xi-\theta) d \eta(\theta) \phi(\xi) d \xi,
\end{aligned}
$$

for $\phi \in C, \psi \in C^{*}=C\left([0,1], \mathbb{R}^{2}\right)$. Then $A\left(\tau_{0}\right)$ and $A^{*}$ are a pair of adjoint operators.

It is not hard to see that $i \tau_{0} \omega_{0}$ is an eigenvalue of $A\left(\tau_{0}\right)$ and $-i \tau_{0} \omega_{0}$ is an eigenvalue of $A^{*}$. Define $q_{1}(\theta)=\left(1, \alpha_{1}\right)^{T} e^{i \omega_{0} \tau_{0} \theta}$ and $q_{2}(\theta)=\overline{q_{1}}(\theta)$; then, one can obtain

$$
A q_{1}(0)=i \omega_{0} \tau_{0} q_{1}(0) .
$$

Then, we have

$$
\alpha_{1}=\frac{\alpha h-i \omega_{0} \tau_{0}}{\alpha \beta}=\alpha_{11}+i \alpha_{12},
$$

where

$$
\begin{aligned}
& \alpha_{11}=\frac{\alpha h}{\alpha \beta}, \\
& \alpha_{12}=-\frac{\omega_{0} \tau_{0}}{\alpha \beta} .
\end{aligned}
$$

Define $q_{1}^{*}\left(\theta^{*}\right)=\left(1, \beta_{1}\right)^{T} e^{-i \omega_{0} \tau_{0} \theta^{*}}$ and $q_{2}^{*}\left(\theta^{*}\right)=\bar{q}_{1}^{*}\left(\theta^{*}\right)$; then, one can obtain

$$
A^{*} q_{1}^{*}(0)=-i \omega_{0} \tau_{0} q_{1}^{*}(0) .
$$

Then, we have

$$
\beta_{1}=\beta_{11}+i \beta_{12}
$$

where

$$
\begin{aligned}
& \beta_{11}=-\frac{\alpha h \cos \omega_{0} \tau_{0}-\sin \omega_{0} \tau_{0} \omega_{0} \tau_{0}}{c_{1}}, \\
& \beta_{12}=-\frac{\omega_{0} \tau_{0} \cos \omega_{0} \tau_{0}+\sin \omega_{0} \tau_{0} \alpha h}{c_{1}} .
\end{aligned}
$$

Let $u(\theta)=\left(u_{1}(\theta), u_{2}(\theta)\right)$ and $v(\theta)=\left(v_{1}(\theta), v_{2}(\theta)\right)^{T}$ with

$$
\begin{aligned}
u_{1}(\theta) & =\frac{q_{1}(\theta)+q_{2}(\theta)}{2} \\
& =\left(\operatorname{Re}\left\{e^{i \omega_{0} \tau_{0} \theta}\right\}, \operatorname{Re}\left\{\alpha_{1} e^{i \omega_{0} \tau_{0} \theta}\right\}\right) \\
& =\left(\begin{array}{c}
\cos \omega_{0} \tau_{0} \theta \\
\alpha_{11} \cos \omega_{0} \tau_{0} \theta-\alpha_{12} \sin \omega_{0} \tau_{0} \theta
\end{array}\right) \\
u_{2}(\theta) & =\frac{q_{1}(\theta)-q_{2}(\theta)}{2 i} \\
& =\left(\lim \left\{e^{i \omega_{0} \tau_{0} \theta}\right\}, \lim \left\{\alpha_{1} e^{i \omega_{0} \tau_{0} \theta}\right\}\right) \\
& =\left(\begin{array}{c}
\sin \omega_{0} \tau_{0} \theta \\
\alpha_{11} \sin \omega_{0} \tau_{0} \theta+\alpha_{12} \cos \omega_{0} \tau_{0} \theta
\end{array}\right)
\end{aligned}
$$

for $\theta \in[-1,0]$ and

$$
\begin{aligned}
v_{1}\left(\theta^{*}\right) & =\frac{q_{1}^{*}\left(\theta^{*}\right)+q_{2}^{*}\left(\theta^{*}\right)}{2} \\
& =\left(\operatorname{Re}\left\{e^{-i \omega_{0} \tau_{0} \theta^{*}}\right\}, \operatorname{Re}\left\{\beta_{1} e^{-i \omega_{0} \tau_{0} \theta^{*}}\right\}\right) \\
& =\left(\begin{array}{c}
\cos \omega_{0} \tau_{0} \theta^{*} \\
\beta_{11} \cos \omega_{0} \tau_{0} \theta^{*}+\beta_{12} \sin \omega_{0} \tau_{0} \theta^{*}
\end{array}\right) \\
v_{2}\left(\theta^{*}\right) & =\frac{q_{1}^{*}\left(\theta^{*}\right)-q_{2}^{*}\left(\theta^{*}\right)}{2 i} \\
& =\left(\lim \left\{e^{-i \omega_{0} \tau_{0} \theta^{*}}\right\}, \lim \left\{\beta_{1} e^{-i \omega_{0} \tau_{0} \theta^{*}}\right\}\right) \\
& =\left(\begin{array}{c}
\sin \omega_{0} \tau_{0} \theta^{*} \\
-\beta_{11} \sin \omega_{0} \tau_{0} \theta^{*}+\beta_{12} \cos \omega_{0} \tau_{0} \theta^{*}
\end{array}\right)
\end{aligned}
$$

for $\theta^{*} \in[-1,0]$.

In the following, we define $\left(u, v^{*}\right)=\left(u_{j}, v^{*}{ }_{k}\right) j, k=1,2$ and construct a new basis $v$

$$
v=\left(v_{1}, v_{2}\right)=\left(v^{*}, u\right)^{-1} v^{*} .
$$

Let $f_{k}=\left(\beta_{k}^{1} \quad \beta_{k}^{2}\right)$ and $c \cdot f_{0}=c_{1} \beta_{0}^{1}+c_{2} \beta_{0}^{2}$ for $c=\left(c_{1}, c_{2}\right)^{T} \in \zeta$; the center space of linear equation (30) is given by

$$
P_{C N} \zeta^{*}=u\left(v,\left\langle\varphi, f_{k}\right\rangle\right) \cdot f_{k} \quad \varphi \in \zeta^{*} .
$$

And $\zeta^{*}=P_{C N} \zeta^{*} \oplus Q$, where $Q$ denotes the complement subspace of $P_{C N} \zeta^{*}$ in $\zeta^{*}$.

As $A\left(\tau_{0}\right)$ is the infinitesimal generator induced by the solution of (32), then (28) can be rewritten as

$$
\dot{U}(t)=A_{\tau_{0}} U(t)+X_{0} F\left(U_{t}, \vartheta\right),
$$

where

$$
X_{0}(\theta)= \begin{cases}0, & -1 \leq \theta<0 \\ I & \theta=0 .\end{cases}
$$

By using the decomposition $\zeta^{*}=P_{C N} \zeta^{*} \oplus Q$ and (45), (30) can be written as

$$
U(t)=U\left(\begin{array}{l}
x_{1}(t) \\
x_{2}(t)
\end{array}\right) \cdot f_{k}+h\left(x_{1}, x_{2}, \vartheta\right),
$$

where $\left(x_{1}(t), x_{2}(t)\right)^{T}=\left(v,\left\langle U(t), f_{k}\right\rangle\right), h\left(x_{1}, x_{2}, \vartheta\right) \in Q$, and $h(0,0,0)=D h(0,0,0)=0$. By using center manifold, we can obtain

$$
U(t)=u\left(\begin{array}{l}
x_{1}(t) \\
x_{2}(t)
\end{array}\right) \cdot f_{k}+h\left(x_{1}, x_{2}, 0\right) .
$$

Let $z=x_{1}-i x_{2}$ and $q_{1}=u_{1}+i u_{2}$, from (48), we can obtain

$$
R(t)=\frac{1}{2}\left(q_{1} z+\bar{q}_{1} \bar{z}\right) \cdot f_{k}+W(z, \bar{z}),
$$


where

$$
\begin{aligned}
W(z, \bar{z}) & =h\left(\frac{(z+\bar{z})}{2}, \frac{i(z-\bar{z})}{2}, 0\right) \\
& \triangleq W_{20}\left(\frac{z^{2}}{2}\right)+W_{11} z \bar{z}+W_{02}\left(\frac{\bar{z}^{2}}{2}\right)+\cdots .
\end{aligned}
$$

Following [18], we have

$$
\dot{z}=i \omega_{0} \tau_{0} z+g(z, \bar{z}),
$$

where

$$
\begin{aligned}
g(z, \bar{z}) & =\left(v_{1}-i v_{2}\right)\left\langle f(U(t), 0), f_{k}\right\rangle \\
& \triangleq g_{20} \frac{z^{2}}{2}+g_{11} z \bar{z}+g_{20} \frac{\bar{z}^{2}}{2}+\cdots
\end{aligned}
$$

for $v(0)=\left(v_{1}(0), v_{2}(0)\right)^{T}$.

By calculation, from (52), we have

$$
\begin{aligned}
\left\langle f(R(t), 0), f_{k}\right\rangle= & \frac{\tau_{0}}{4} \frac{1}{\pi} \int_{0}^{\pi} \cos ^{3} k x d x\left(\begin{array}{l}
d_{11} \\
d_{21}
\end{array}\right) \frac{z^{2}}{2} \\
& +\frac{\tau_{0}}{4} \frac{1}{\pi} \int_{0}^{\pi} \cos ^{3} k x d x\left(\begin{array}{l}
d_{12} \\
d_{22}
\end{array}\right) z \bar{z} \\
& +\frac{\tau_{0}}{4} \frac{1}{\pi} \int_{0}^{\pi} \cos ^{3} k x d x\left(\begin{array}{l}
d_{13} \\
d_{23}
\end{array}\right) \frac{\bar{z}^{2}}{2} \\
& +\frac{\tau_{0}}{2}\left(\begin{array}{l}
d_{14} \\
d_{24}
\end{array}\right) \frac{z^{2} \bar{z}}{2},
\end{aligned}
$$

where

$$
\begin{aligned}
d_{11} & =a c_{2}, \\
d_{12} & =c_{2} e^{-i \omega_{0} \tau_{0}}, \\
d_{21} & =a c_{2}, \\
d_{22} & =c_{2} e^{-i \omega_{0} \tau_{0}} \\
d_{31} & =a c_{2}, \\
d_{32} & =c_{2} e^{-i \omega_{0} \tau_{0}} \\
d_{41} & =a c_{2}\left\langle\left(2 W_{11}^{(1)}(0)+W_{20}^{(1)}(0)\right) \cos k x, \cos k x\right\rangle \\
& +\frac{1}{4} a c_{3} \frac{1}{\pi} \int_{0}^{\pi} \cos { }^{4} k x d x \\
d_{42} & =c_{2}\left\langle\left(2 W_{11}^{(1)}(0) e^{-i \omega_{0} \tau_{0}}+W_{20}^{(1)}(0) e^{i \omega_{0} \tau_{0}}\right)\right. \\
\cdot & \cos k x, \cos k x\rangle+\frac{1}{4} c_{3} \frac{1}{\pi} \int_{0}^{\pi} \cos ^{4} k x d x \\
\left\langle W_{i j}^{n}(\theta), \cos k x\right\rangle=\frac{1}{\pi} \int_{0}^{\pi} W_{i j}^{n}(\theta)(x) d x, & i+j=2, n=1,2,3,4 .
\end{aligned}
$$

It is easy to see $(1 / \pi) \int_{0}^{\pi} \cos ^{3} k x d x=0$ when $k \neq 0$. Let $\left(\rho_{1}, \rho_{2}\right)=v_{1}(0)-i v_{2}(0)$; compare the coefficients with (52); we can obtain

$$
\begin{aligned}
& g_{20}= \begin{cases}0 & k=\mathbb{N} \\
\frac{\tau}{4}\left(d_{11} \rho_{1}+d_{21} \rho_{2}\right) & k=0\end{cases} \\
& g_{11}= \begin{cases}0 & k=\mathbb{N} \\
\frac{\tau}{4}\left(d_{12} \rho_{1}+d_{22} \rho_{2}\right) & k=0\end{cases}
\end{aligned}
$$

$$
\begin{aligned}
& g_{02}= \begin{cases}0 & k=\mathbb{N} \\
\frac{\tau}{4}\left(d_{13} \rho_{1}+d_{23} \rho_{2}\right) & k=0 .\end{cases} \\
& g_{21}=\frac{\tau}{2}\left(d_{14} \rho_{1}+d_{24} \rho_{2}\right)
\end{aligned}
$$

Because of $W_{20}(\theta)$ and $W_{11}(\theta)$ in the $g_{21}$, we need to determine them. It follows from (52) that

$$
\begin{aligned}
\dot{W}(z, \bar{z})= & W_{20} z \dot{z}+W_{11} \dot{z} \bar{z}+W_{11} z \dot{\bar{z}}+W_{02} \frac{\dot{z z}}{} \\
& +\cdots, \\
A_{\tau_{0}} \dot{W}(z, \bar{z})= & A_{\tau_{0}} W_{20} \frac{z^{2}}{2}+A_{\tau_{0}} W_{11} z \bar{z}+A_{\tau_{0}} W_{02} \frac{\bar{z}^{2}}{2} \\
& +\cdots .
\end{aligned}
$$

According to [18], we have

$$
\dot{W}=A_{\tau_{0}} W+H(z, \bar{z}),
$$

where

$$
\begin{aligned}
H(z, \bar{z})= & H_{20} \frac{z^{2}}{2}+H_{11} z \bar{z}+H_{02} \frac{\bar{z}^{2}}{2}+\cdots \\
= & X_{0} f(U(t), 0) \\
& -u\left(v,\left\langle X_{0} f(U(t), 0), f_{k}\right\rangle\right) \cdot f_{k} .
\end{aligned}
$$

By comparing (56), (58), and (59), we have

$$
\begin{aligned}
\left(A_{\tau_{0}}-2 i \omega_{0} \tau_{0}\right) W_{20}(\theta) & =-H_{20}(\theta), \\
A_{\tau_{0}} W_{11}(\theta) & =-H_{11}(\theta) .
\end{aligned}
$$

Expanding (59), we can obtain

$$
\begin{aligned}
H(z, \bar{z})= & -\frac{1}{2}\left[q_{1}(\theta) g_{20}+q_{2}(\theta) \bar{g}_{02}\right] \cdot f_{k} \frac{z^{2}}{2} \\
& -\frac{1}{2}\left[q_{1}(\theta) g_{11}+q_{2}(\theta) \bar{g}_{11}\right] \cdot f_{k} z \bar{z}+\cdots .
\end{aligned}
$$

According to (60), the following can be obtained:

$$
\begin{aligned}
& H_{20}(\theta)= \begin{cases}0 & k \in \mathbb{N} \\
-\frac{1}{2}\left[q_{1}(\theta) g_{20}+q_{2} g_{11}(\theta)\right] \cdot f_{0} & k=0\end{cases} \\
& H_{11}(\theta)= \begin{cases}0 & k \in \mathbb{N} \\
-\frac{1}{2}\left[q_{1}(\theta) g_{11}+q_{2} \bar{g}_{11}(\theta)\right] \cdot f_{0} & k=0 .\end{cases}
\end{aligned}
$$


According to (60), (62), and $q_{1}(\theta)=q_{1}(0) e^{i \omega_{0} \tau_{0}}$, we can obtain

$$
\begin{aligned}
W_{20}(\theta)= & \frac{1}{2}\left[\frac{i g_{20}}{\omega_{0} \tau_{0}} q_{1}(\theta)+\frac{i \bar{g}_{20}}{3 \omega_{0} \tau_{0}} q_{2}(\theta)\right] \cdot f_{k} \\
& +E_{1} e^{2 i \omega_{0} \tau_{0} \theta} \\
W_{11}(\theta)= & \frac{1}{2}\left[\frac{-i g_{11}}{\omega_{0} \tau_{0}} q_{1}(\theta)+\frac{i \bar{g}_{11}}{\omega_{0} \tau_{0}} q_{2}(\theta)\right] \cdot f_{k}+E_{2},
\end{aligned}
$$

where $E_{1}=\left(E_{1}^{(1)}, E_{1}^{(2)}\right) \in \mathfrak{R}^{2}, E_{2}=\left(E_{2}^{(1)}, E_{2}^{(2)}\right) \in \mathfrak{R}^{2}$, and $E_{1}, E_{2}$ are constant vector.

In order to seek appropriate $E_{1}, E_{2}$, we can obtain the following by the definition of $A_{\tau_{0}}$ and (60)

$$
\begin{aligned}
H_{20}(0)= & 2 i \omega_{0} \tau_{0} W_{20}(0)-\tau_{0} D \Delta W_{20}(0) \\
& -L\left(\tau_{0}\right) W_{20}(\theta) \\
H_{11}(0)= & -\tau_{0} D \Delta W_{11}(0)-L\left(\tau_{0}\right) W_{11}(\theta),
\end{aligned}
$$

where

$$
\begin{aligned}
& H_{20}(0) \\
& = \begin{cases}\frac{\tau_{0}}{4}\left(d_{11}, d_{21}\right)^{T} \cos ^{2} k x & k \in \mathbb{N} \\
\frac{\tau_{0}}{4}\left(d_{11}, d_{21}\right)^{T}-\frac{1}{2}\left[q_{1}(0) g_{20}+q_{2}(0) \bar{g}_{02}\right] \cdot f_{k} & k=0\end{cases} \\
& H_{11}(0) \\
& = \begin{cases}\frac{\tau_{0}}{4}\left(d_{12}, d_{22}\right)^{T} \cos ^{2} k x & k \in \mathbb{N} \\
\frac{\tau_{0}}{4}\left(d_{12}, d_{22}\right)^{T}-\frac{1}{2}\left[q_{1}(0) g_{11}+q_{2}(0) \bar{g}_{11}\right] \cdot f_{k} & k=0 .\end{cases}
\end{aligned}
$$

Then, we can obtain

$$
\begin{aligned}
& E_{1} \\
& \left.\qquad \begin{array}{ll}
4 & \frac{1}{2 i \omega_{0}+d_{1} k^{2}-\alpha\left(c_{1}-\gamma\right)} \\
-c_{1} e^{-2 i \omega_{0} \tau} & 2 i \omega_{0}+d_{2} k^{2}+(q+\beta)
\end{array}\right)^{-1} \\
& \cdot\left(\begin{array}{l}
d_{11} \\
d_{21}
\end{array}\right) \cos ^{2} k x \\
& E_{2}=\frac{1}{4}\left(\begin{array}{cc}
d_{1} k^{2}-\alpha\left(c_{1}-\gamma\right) & \alpha \beta \\
-c_{1} & d_{2} k^{2}+(q+\beta)
\end{array}\right)^{-1} \\
& \quad \cdot\left(\begin{array}{l}
d_{11} \\
d_{21}
\end{array}\right) \cos ^{2} k x .
\end{aligned}
$$

Now, we can calculate $W_{20}(\theta)$ and $W_{11}(\theta) ; g_{21}$ is also expressed, and then the following important parameter can be obtained

$$
\begin{aligned}
C_{1}(0) & =\frac{g_{21}}{2}, \\
\mu_{2} & =-\frac{\operatorname{Re}\left\{C_{1}(0)\right\}}{\operatorname{Re}\left\{\lambda^{\prime}\left(\tau_{0}\right)\right\}}, \\
\beta_{2} & =2 \operatorname{Re}\left\{C_{1}(0)\right\}, \\
T_{2} & =-\frac{\operatorname{Im}\left\{C_{1}(0)\right\}+\mu_{2} \operatorname{Im}\left\{\lambda^{\prime}\left(\tau_{0}\right)\right\}}{\omega_{0} \tau_{0}} .
\end{aligned}
$$

Theorem 6. By Theorem 4, one has the following results:

(i) The sign of $\mu_{2}$ can determine the direction of Hopf bifurcation: if $\mu_{2}>0\left(\mu_{2}<0\right)$, the Hopf bifurcation is supercritical (subcritical) and the bifurcating periodic solution exists for $\tau>\tau_{0}\left(\tau<\tau_{0}\right)$.

(ii) The sign of $\beta_{2}$ determines the stability of the bifurcating periodic solutions: if $\beta_{2}<0,\left(\beta_{2}>0\right)$ the bifurcation periodic solutions are stable (unstable).

(iii) The sign of $T_{2}$ determines the period of the bifurcating periodic solutions: if $T_{2}>0\left(T_{2}<0\right)$ the period increases (decreases).

\section{Numerical Simulations}

In this section, two simulations are given to validate the obtained theorems. Let $d_{1}=d_{2}=0.1, \alpha=3, \beta=0.2$, $\gamma=0.25, q=0.05, i(Y(t, x))=e^{Y(t, x)} /\left(1+e^{Y(t, x)}\right)$, and system (2) can be rewrite as follows:

$$
\begin{aligned}
& \frac{\partial Y(t, x)}{\partial t} \\
& =0.1 * \Delta Y(t, x) \\
& \quad+3\left(\frac{e^{Y(t, x)}}{1+e^{Y(t, x)}}-0.2 Y(t, x)-0.25 K(t, x)\right)
\end{aligned}
$$

$$
\begin{aligned}
& \frac{\partial K(t, x)}{\partial t} \\
& \quad=0.1 * \Delta K(t, x)+\frac{e^{Y(t, x)}}{1+e^{Y(t, x)}}-0.25 K(t, x),
\end{aligned}
$$

the Neumann boundary conditions of system (2) is as follows:

$$
\frac{\partial u_{1}}{\partial x}=\frac{\partial v_{1}}{\partial x}=\frac{\partial u_{2}}{\partial x}=\frac{\partial v_{2}}{\partial x}=0, \quad t>0, x \in \partial \Omega,
$$

and the initial conditions is as follows:

$$
u_{1}(t, x)=v_{1}(t, x)=u_{2}(t, x)=v_{2}(t, x)=0.1 \text {. }
$$

By simple calculations, we can obtain the equilibrium is $(0.498,2.489), c_{1}<\gamma\left(\gamma-c_{1}\right)(q+\beta)-\left(\beta c_{1}\right)=-0.0198<$ $0, \omega_{0}=0.366$, and $\tau_{0} \approx 2.3805$. Consider $\tau=2.2<\tau_{0}$, by Theorem 4, system (2) is asymptotically stable, which is verified in Figures 1 and 2.

Consider $\tau=2.6>\tau_{0}$; by Theorem 4 , system (2) is unstable and a Hopf bifurcation occurs. By simple calculation, we can obtain the parameter $C_{1}(0)=7.0694-0.892020 i, \mu_{2}=$ $7.3686>0$, and $\beta_{2}=14.1388>0$ which means Hopf bifurcation is supercritical since $\mu_{2}>0$. The simulations are shown in Figures 3 and 4, which indicate that there is a stable limit cycle.

In the following, we investigate the effect of diffusion on the dynamics of system (2). Let $d_{i}=0.1,0.5$ and $\tau=2.8>\tau_{0}$; the diagrams are shown in Figures 4-7. From Figure 4, we can see that system (2) have a periodic solution when $d_{i}=0.1$. With $D$ increasing to 0.5 , the periodic solution disappear, 


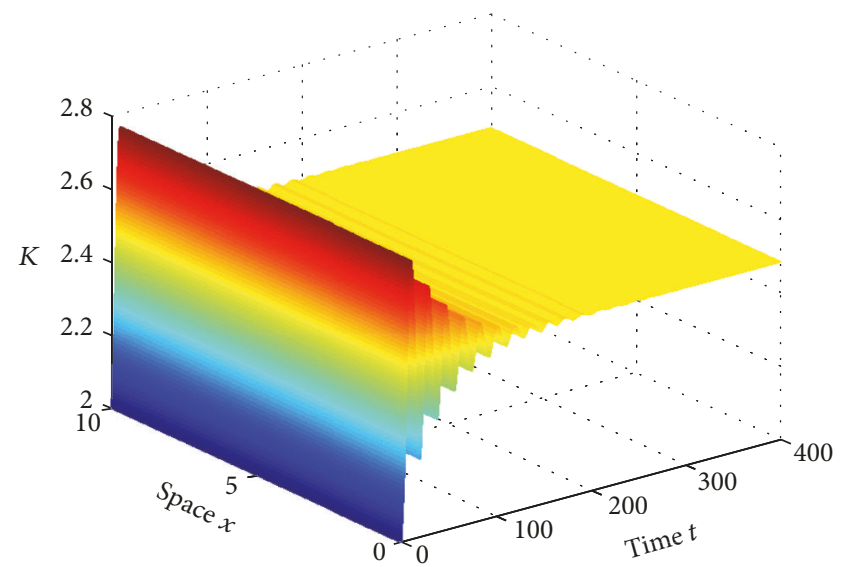

(a)

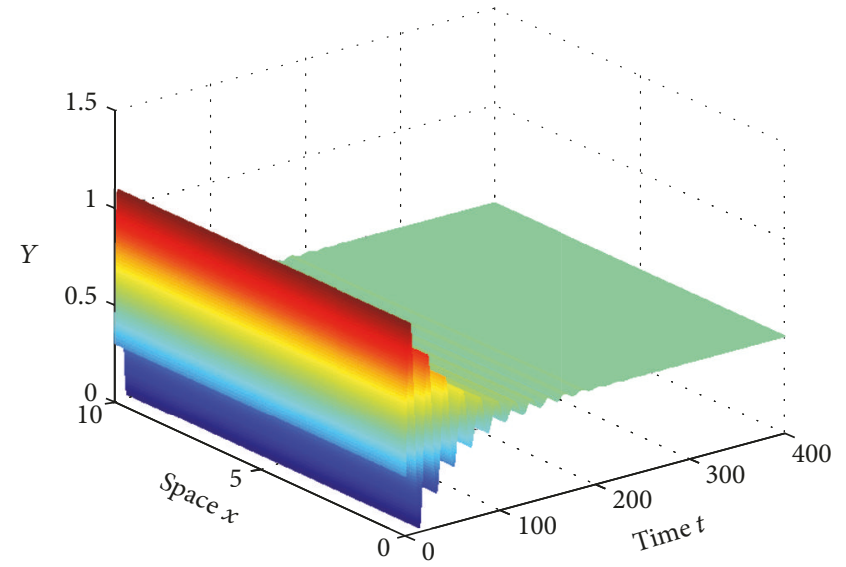

(b)

FIgURE 1: The temporal solution of system (2) with $\tau=2.2$.

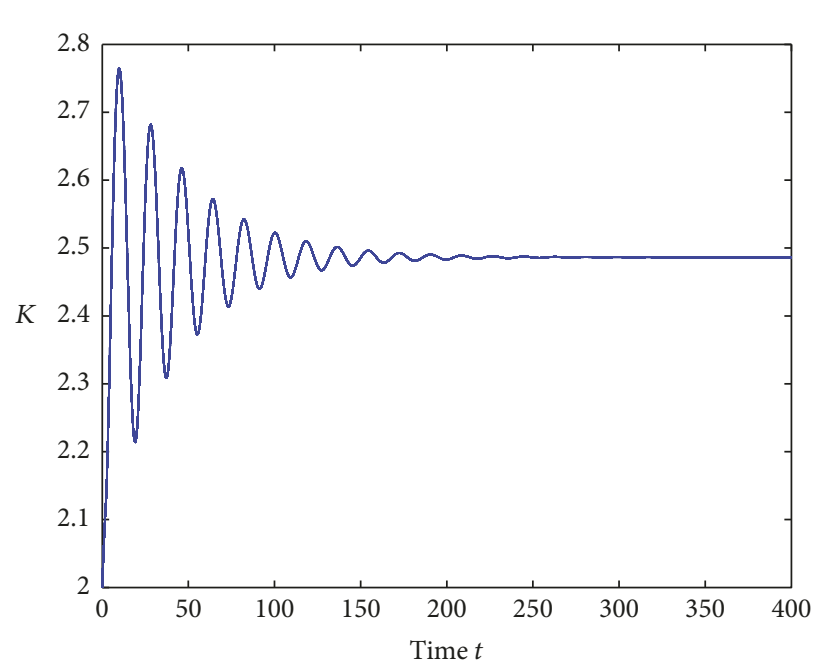

(a)

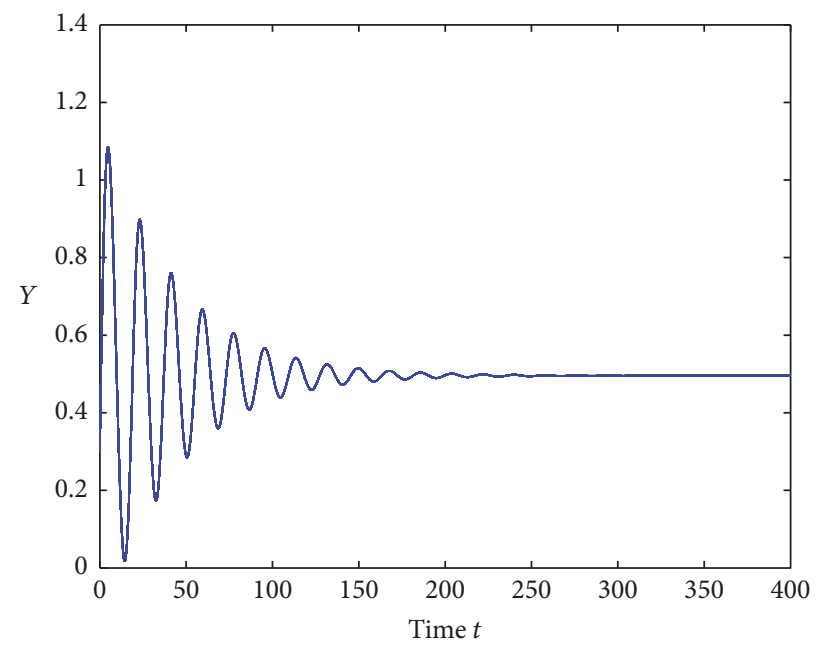

(b)

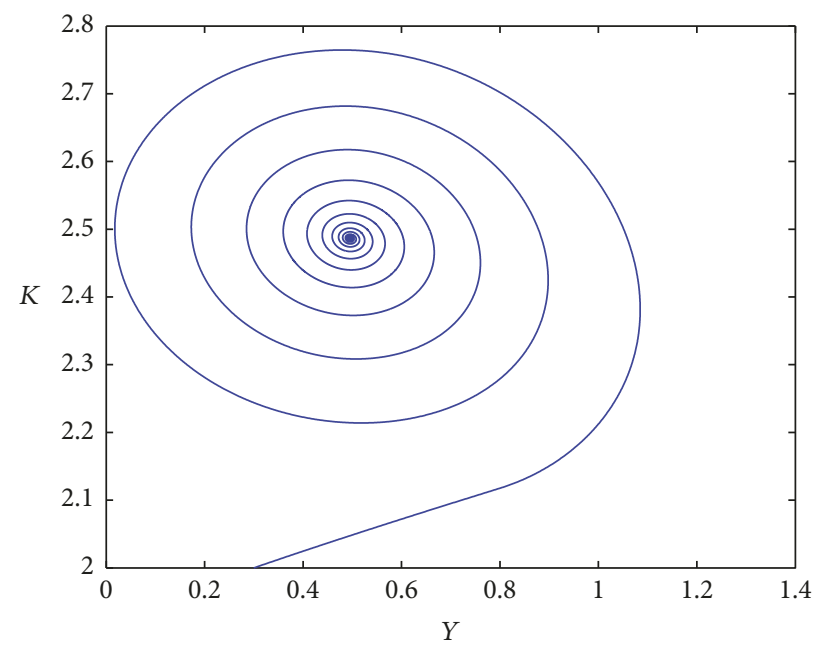

(c)

Figure 2: The temporal solution of system (2) with $\tau=2.2, x=5$. 


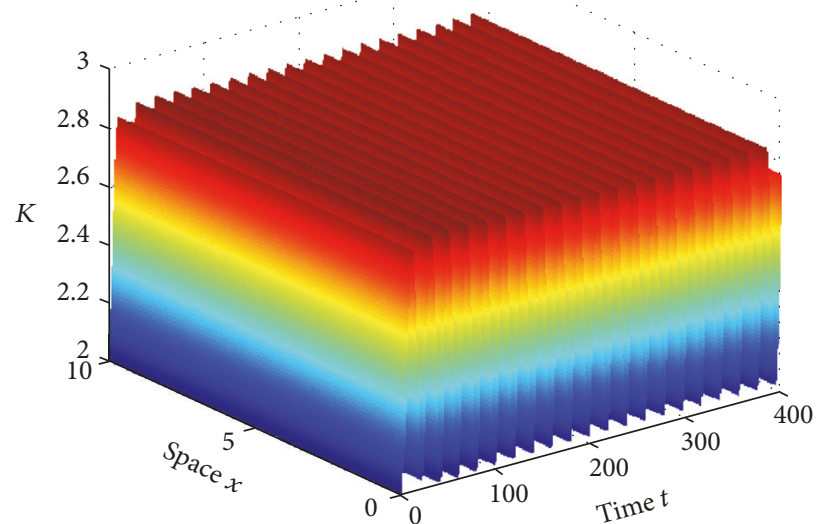

(a)

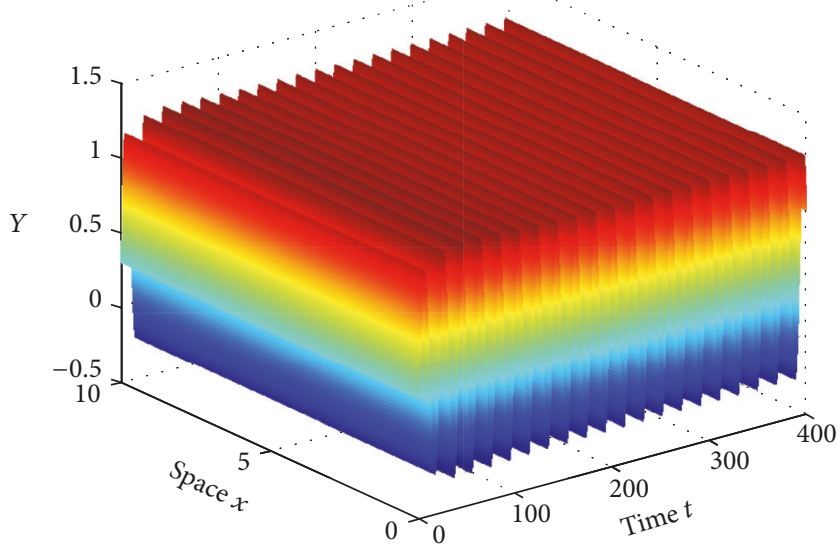

(b)

FIGURE 3: The periodic solutions diagram of system (2) with $\tau=2.6$.

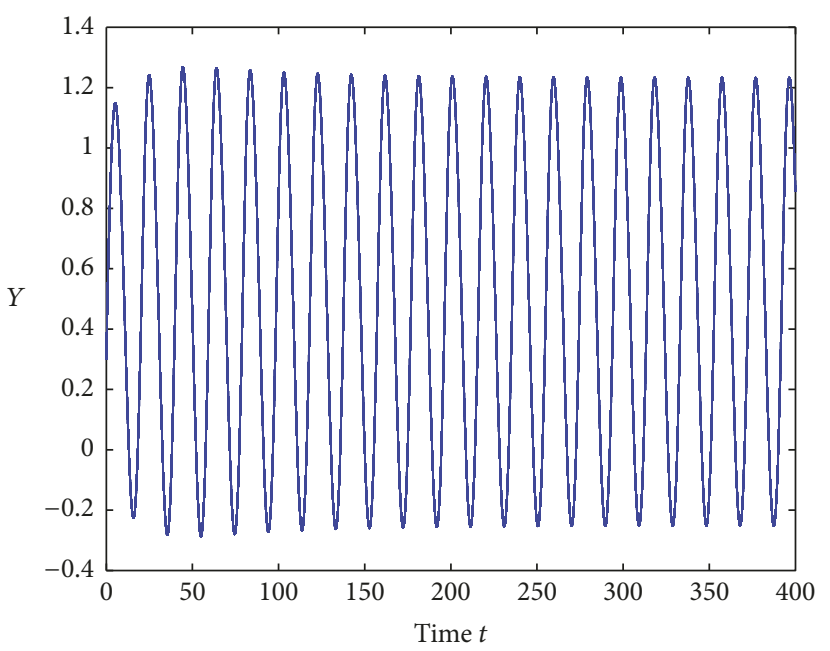

(a)

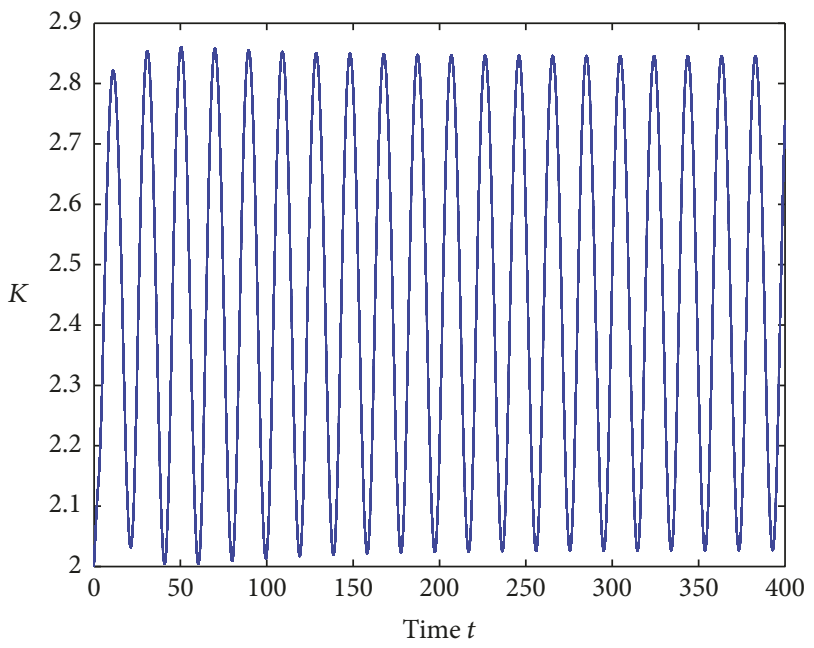

(b)

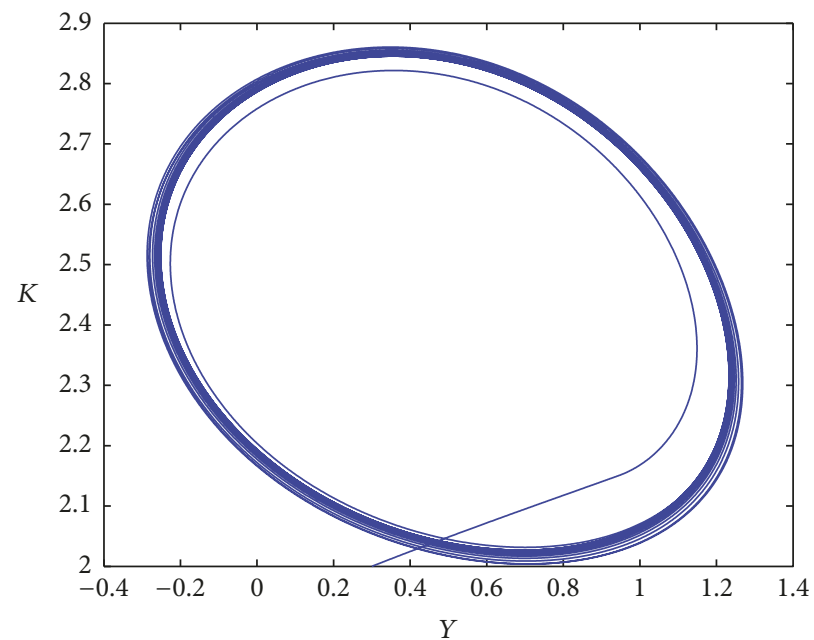

(c)

FIgURE 4: The periodic solutions diagram of system (2) with $\tau=2.6, x=5$. 


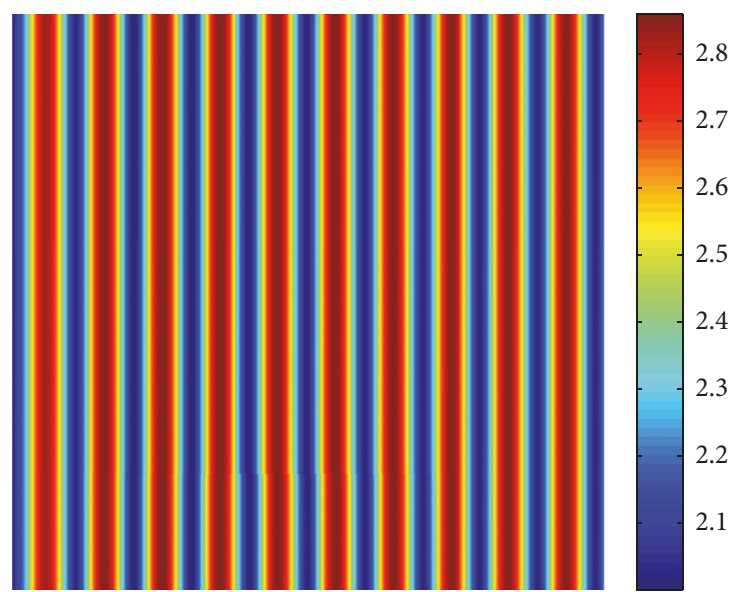

FIGURE 5: The spatial pattern of system (2) with $\tau=2.6, d_{i}=0.1$.

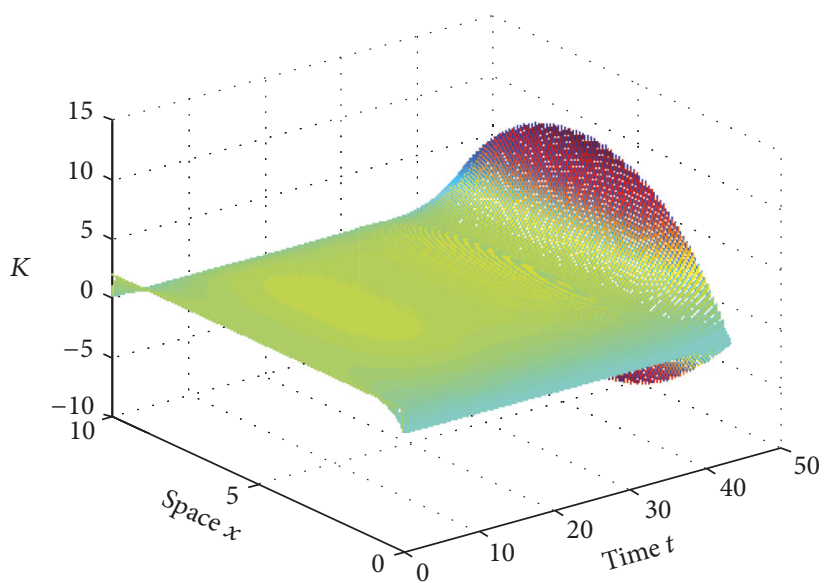

(a)

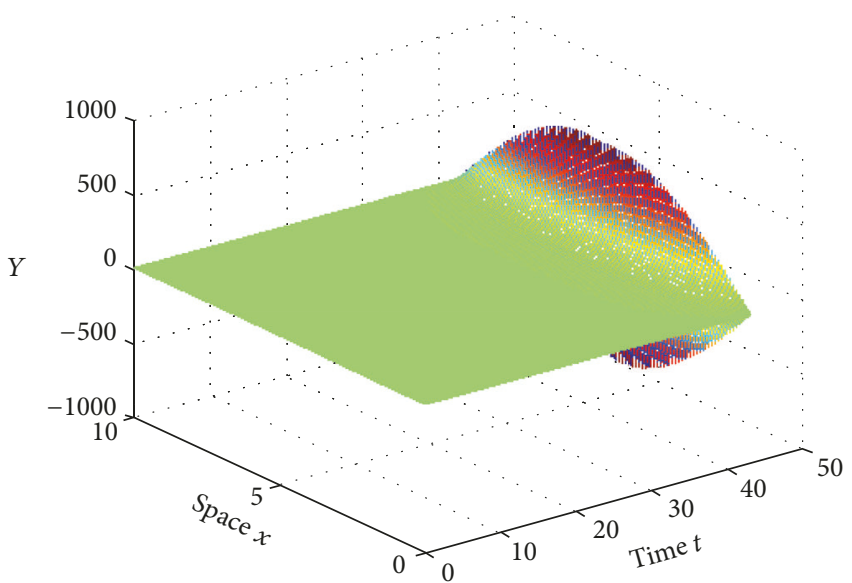

(b)

FIGURE 6: The temporal solution of system (2) with $\tau=2.6, d_{i}=0.5$.
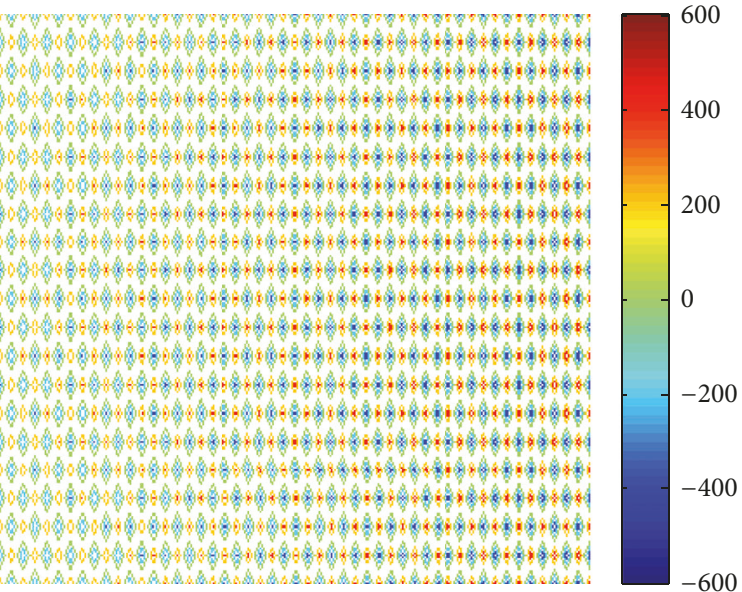

FIGURE 7: The spatial pattern of system (2) with $\tau=2.6, d_{i}=0.5$. 
system (2) becomes divergent, which is shown in Figure 6. Moreover, from Figures 5-7, we can see that the spatial pattern with $d_{i}=0.1$ is different from the spatial pattern with $d_{i}=0.5$. To summarize, it can be seen the diffusion coefficient affects the pattern formation of system (2).

\section{Conclusions}

It is well known that diffusion effects of economic activities are widespread phenomenon that existed all over the world. As a result of the impact of the growth pole, the diffusion effects are the main interactions in economic activities. So, the diffusion effect should be considered in the business cycle model. In this paper, we consider a Kaldor-Kalecki business cycle model with diffusion effect and time delay under the Neumann boundary conditions. First the time-independent and time-dependent stability are investigated. Then, we find that the time delay can give rise to the Hopf bifurcation when the time delay passes a critical value. Moreover, the normal form of Hopf bifurcation is obtained. Finally, numerical results not only validate the obtained theorems, but also show that the diffusion coefficients play a key role in the spatial pattern. With the diffusion coefficients increasing, different patterns appear.

\section{Conflicts of Interest}

The authors declare that they have no conflicts of interest.

\section{Acknowledgments}

This work was supported in part by the National Natural Science Foundation of China under Grant 61503310, in part by the Fundamental Research Funds for the Central Universities under Grants 2017CDJSK02XK24, 2017CDJKY, and 106112015CDJSK02JD06, in part by China Postdoctoral Foundation 2016M600720, and in part by Chongqing Postdoctoral Project under Grant Xm2016003.

\section{References}

[1] R. Rajaram, B. Castellani, and A. N. Wilson, "Advancing shannon entropy for measuring diversity in systems," Complexity, vol. 2017, Article ID 8715605, 10 pages, 2017.

[2] D. Wollmann and M. T. A. Steiner, "The strategic decisionmaking as a complex adaptive system: a conceptual scientific model," Complexity, vol. 2017, Article ID 7954289, 13 pages, 2017.

[3] H. Saijo, "The uncertainty multiplier and business cycles," Journal of Economic Dynamics and Control, vol. 78, pp. 1-25, 2017.

[4] K. Hattaf, D. Riad, and N. Yousfi, "A generalized business cycle model with delays in gross product and capital stock," Chaos, Solitons \& Fractals, vol. 98, pp. 31-37, 2017.

[5] S. Yildırım-Karaman, "Uncertainty in financial markets and business cycles," Economic Modelling, vol. 68, pp. 329-339, 2018.

[6] R. A. K. Cox, A. Dayanandan, H. Donker, and J. Nofsinger, "The Bad, the boom and the bust: Profit warnings over the business cycle," Journal of Economics and Business, vol. 89, pp. 13-19, 2017.
[7] S. Yıldırım-Karaman, "Uncertainty shocks, central bank characteristics and business cycles," Economic Systems, vol. 41, no. 3, pp. 379-388, 2017.

[8] B. Zeng, G. Chen, and S.-f. Liu, "A novel interval grey prediction model considering uncertain information," Journal of The Franklin Institute, vol. 350, no. 10, pp. 3400-3416, 2013.

[9] A. Kaddar and H. Talibi Alaoui, "Hopf bifurcation analysis in a delayed Kaldor-Kalecki model of business cycle," Nonlinear Analysis: Modelling and Control, vol. 13, no. 4, pp. 439-449, 2008.

[10] X. P. Wu, "Codimension-2 bifurcations of the Kaldor model of business cycle," Chaos, Solitons and Fractals, vol. 44, no. 1-3, pp. 28-42, 2011.

[11] X. P. Wu and L. Wang, "Multi-parameter bifurcations of the KALdor-KALecki model of business cycles with delay," Nonlinear Analysis: Real World Applications, vol. 11, no. 2, pp. 869-887, 2010.

[12] D. Riad, K. Hattaf, and N. Yousfi, "Dynamics of a delayed business cycle model with general investment function," Chaos, Solitons and Fractals, vol. 85, pp. 110-119, 2016.

[13] X. P. Wu, "Zero-Hopf bifurcation analysis of a KALdorKALecki model of business cycle with delay," Nonlinear Analysis: Real World Applications, vol. 13, no. 2, pp. 736-754, 2012.

[14] L. I. Dobrescu and D. Opris, "Neimark-Sacker bifurcation for the discrete-delay Kaldor-Kalecki model," Chaos, Solitons \& Fractals, vol. 41, no. 5, pp. 2405-2413, 2009.

[15] J. Yu and M. Peng, "Stability and bifurcation analysis for the Kaldor-Kalecki model with a discrete delay and a distributed delay," Physica A: Statistical Mechanics and its Applications, vol. 460, pp. 66-75, 2016.

[16] T. Dong, W. Xu, and X. Liao, "Hopf bifurcation analysis of reaction-diffusion neural oscillator system with excitatory-toinhibitory connection and time delay," Nonlinear Dynamics, vol. 89, no. 4, pp. 2329-2345, 2017.

[17] S. G. Ruan and J. J. Wei, "On the zeros of transcendental functions with applications to stability of delay differential equations with two delays," Dynamics of Continuous, Discrete \& Impulsive Systems A: Mathematical Analysis, vol. 10, no. 6, pp. 863-874, 2003.

[18] J. Wu, Theory and applications of partial functional differential equations, Springer Science \& Business Media, 2012. 


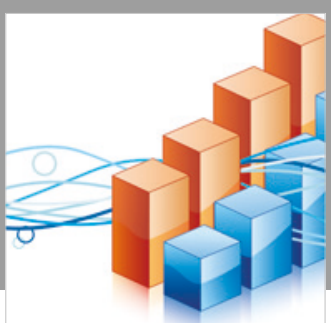

Advances in

Operations Research

\section{-n-m}
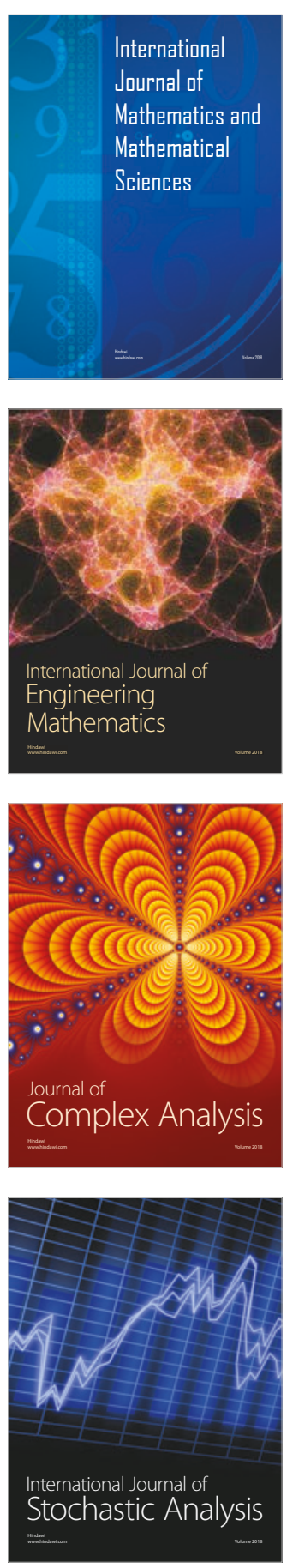
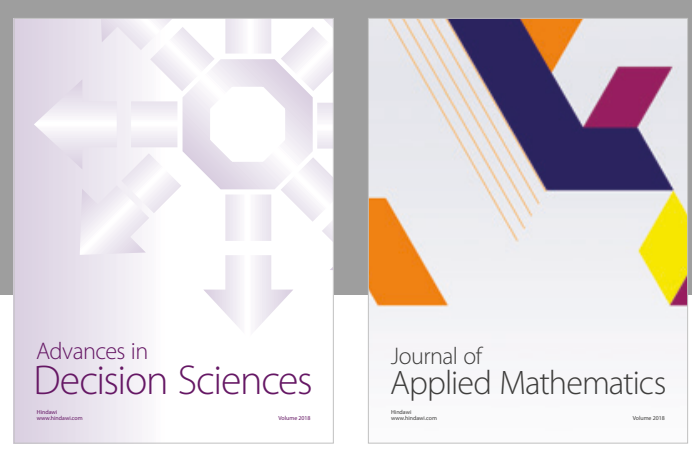

Journal of

Applied Mathematics
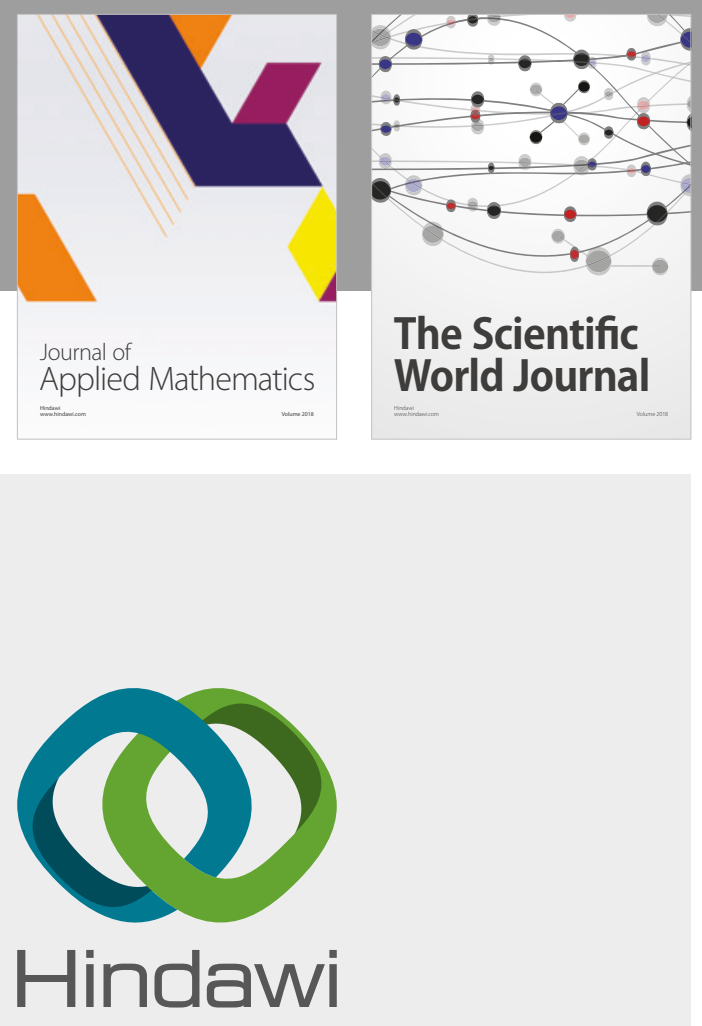

Submit your manuscripts at

www.hindawi.com

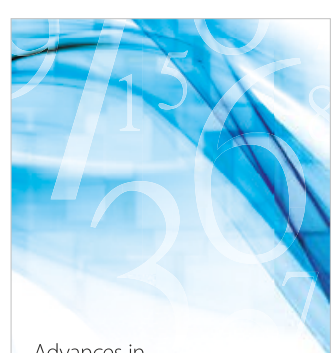

Advances in
Numerical Analysis
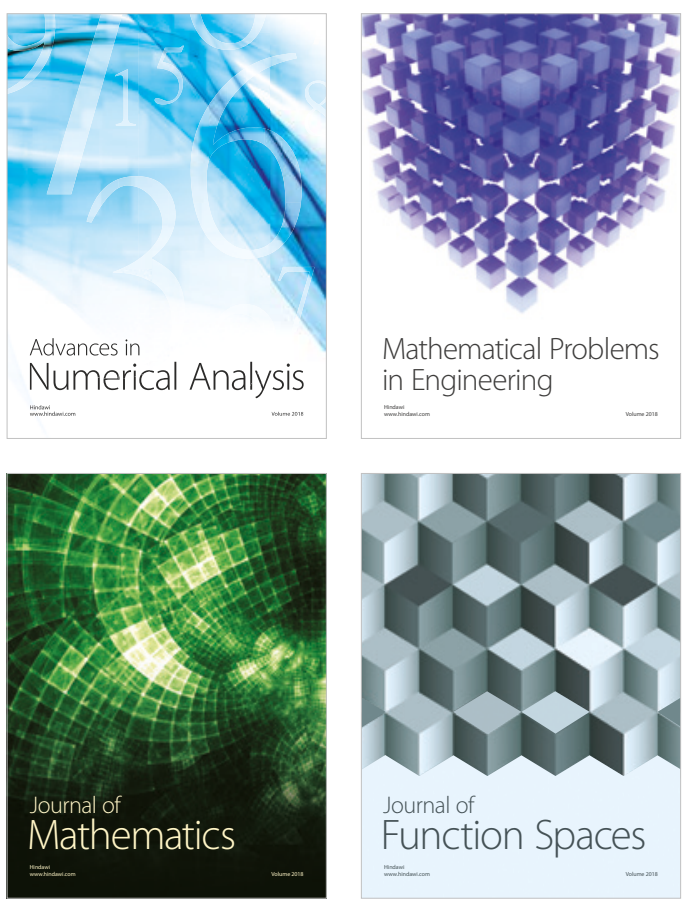

Mathematical Problems in Engineering

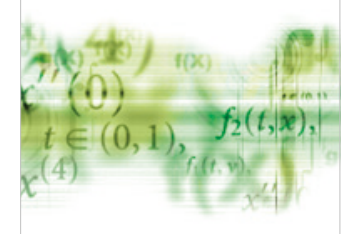

International Journal of

Differential Equations

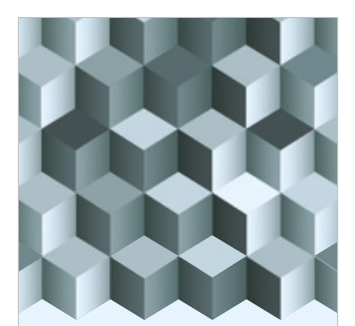

Journal of

Function Spaces
The Scientific

World Journal

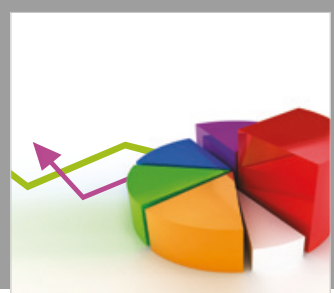

Journal of

Probability and Statistics
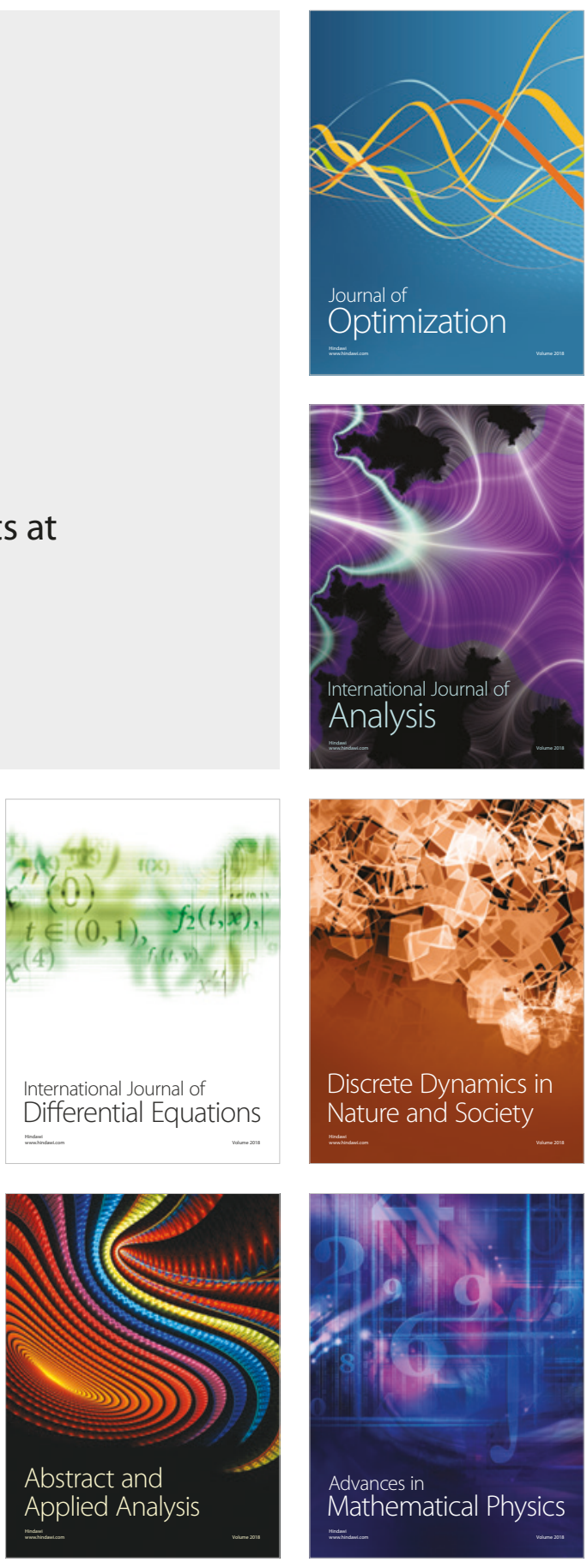